\title{
MODELOS \\ COMPUTACIONALES Y ANÁLISIS DE LA POLÍTICA ECONÓMICA EN COLOMBIA
}

\author{
Andres Escobar-Espinoza \\ Universidad de Cartagena \\ Colombia
}

Diego Alejandro Guevara-Castañeda Universidad Nacional de Colombia Colombia

Miguel Andrés Uribe Veloza New York University Estados Unidos 
Panorama Económico, Vol. 25 - No. 4 (Octubre - Diciembre de 2017), pp. 535-558

Andres Escobar-Espinoza

Diego Alejandro Guevara-Castañeda

Miguel Andrés Uribe Veloza

\title{
Modelos Computacionales y Análisis de la Política Económica en Colombia
}

\section{Resumen}

Este artículo presenta una breve revisión de los modelos computacionales macroeconómicos que se han utilizado en Colombia y sus principales características. Primero, se realiza una revisión de los principales modelos de Equilibrio General Computable (EGC) implementados para el análisis de la política económica identificando sus principales características teóricas. Adicionalmente, se revisan algunos modelos de Equilibrio General Dinámico Estocástico desarrollados en Colombia. Posteriormente, se presenta el enfoque de los modelos Post-Keynesianos Stock-Flujo Consistentes (SFC), identificando algunas diferencias y puntos relevantes respecto a los modelos anteriores. De esta forma, se busca mostrar la pertinencia de utilizar modelos macroeconomicos de corte heterodoxo como herramienta adicional para examinar de manera lógica y coherente las interrelaciones de los sectores real y financiero, además de resaltar su importancia para establecer e identificar aquellos procesos insostenibles que pueden generar periodos de crisis y cambios estructurales no deseados en las economías.

Palabras clave: Modelos computacionales, Equilibrio general computable, Política económica, Modelos Stock-Flow Consistent.

Clasificación JEL: C60, C63, C65, C69, E17, E61

\section{Computational models and Economic Policy Analysis in Colombia}

\begin{abstract}
This research presents a revision of macroeconomic computational models implemented in Colombia and their main characteristics. First, the main Computable General Equilibrium (CGE) Models develoed for economic policy analysis are presented including their main theoretical assumptions. In addition, the new Dynamic Stochastic General Equilibrium (DSGE) Models developed for policy analysis are briefly discussed. Finally, the so called Stock Flow Consistent approach under the PostKeynesian tradition is presented, highlighting its main differences to orthodox applied macroeconomic models discussed earlier. By this, the relevance of applied heterodox macroeconomic models is discussed as an additional tool for macroeconomic policy analysis, in a coherent and logic framework, the interrelations betwen the real and financial sectors of any economy, and to identify unsustainable processes that may lead to economic crisis and unwanted structural changes
\end{abstract}

Keywords: Computational models, Computable General Equilibrium Models, Economic Policy, Stock Flow Consistent Models.

JEL Classification: C60, C63, C65, C69, E17, E61

\section{Modèles de Calcul et Analyse de la Politique Économique en Colombie}

\section{Résumé}

Cette recherche présente une révision des modèles de calcul macroéconomiques mis en œuvre en Colombie et de leurs principales caractéristiques. Tout d'abord, les principaux modèles d'équilibre général calculable (CGE) développés pour l'analyse de politique économique sont présentés, y compris leurs principales hypothèses théoriques. En outre, les nouveaux modèles d'équilibre stochastique dynamique (DSGE) développés pour l'analyse des politiques sont brièvement abordés. Enfin, l'approche dite de la cohérence des flux de stock selon la tradition post-keynésienne est présentée, soulignant ses principales différences avec les modèles macroéconomiques appliqués orthodoxes discutés précédemment. Ainsi, la pertinence des modèles macroéconomiques hétérodoxes appliqués est examinée en tant qu'outil supplémentaire pour l'analyse des politiques macroéconomiques, dans un cadre cohérent et logique, les interrelations entre les secteurs réel et financier de toute économie et pour identifier les processus non durables susceptibles crise et changements structurels indésirables.

Mots-clés: Modèles de calcul, Modèles d'équilibre général calculables, Politique économique, Modèles compatibles avec les mouvements des stocks.

Nomenclature JEL: C60, C63, C65, C69, E17, E61 


\section{Modelos Computacionales y Análisis de la Política Económica en Colombia}

INFORMACIÓN DEL ARTÍCULO

Recepción de artículo: 10/07/2017

Concepto de evaluación: 17/08/2017

Aceptación de artículo: 30/09/2017
Andres Escobar-Espinoza* Universidad de Cartagena, Colombia

Diego Alejandro Guevara-Castañeda Universidad Nacional de Colombia, Colombia

Miguel Andrés Uribe Veloza New York University, Estados Unidos

\section{INTRODUCCIÓN}

Desde la década de los sesenta en Colombia se han implementado una serie de modelos macroeconómicos con la finalidad de establecer los posibles impactos económicos de ciertas políticas. Si bien es cierto que las decisiones de política económica no siempre se han tomado en base a estas simulaciones económicas, los modelos CGE (Computable General Equilibrium) se han constituido en el instrumento por excelencia para la comprensión de muchas de ellas. Estos modelos, inspirados en los trabajos de Wassily Leontief (1937) y Leif Johansen (1960), han presentado distintas características para el caso colombiano, y que, a pesar de su flexibilidad al momento de introducir distintos enfoques teóricos, se han caracterizado por su orientación neoclásica y por tratar solamente el sector real de la economía. Incluso han surgido aplicaciones de los más recientes y sofisticados modelos DSGE (Dynamic Stochastic General Equilibrium), de corte microfundamentado y neoclásico, los cuales aún insisten en el sector real. Por consiguiente, estas particularidades han determinado los resultados de los análisis de políticas sin enfatizar y profundizar en el funcionamiento del sector financiero de la economía.

Sin embargo, y siguiendo la tradición teórica Keynesiana, emerge el enfoque de simulación macroeconómica SFC (Stock-Flow Consistent) inspirado en los trabajos de Wynne Godley y Francis Cripps (1983), y que se constituye como elemento integrador del pensamiento post-Keynesiano moderno. Esta aproximación se caracteriza por considerar de manera consistente la interrelación de los sectores real y monetario de la economía en un mismo modelo, teniendo en consideración la importancia del capital tangible y

\footnotetext{
* Autor para correspondencia

Correos electrónicos: aescobare@unicartagena.edu.co*,dieguevarac@unal.edu.co, muv200@nyu.edu
} 
del capital financiero. Donde éste último, ha tomado una mayor importancia en la últimas tres décadas, y por tanto, no puede ser relegado del modelamiento a manera de "simplificación", puesto que limita fuertemente las posibilidades descriptivas del modelo dada la relevancia del sector financiero en esta etapa de capitalismo globalizado.

El reconocimiento de estrategias de modelamiento de diversas corrientes de pensamiento económico es fundamental ante la complejidad inherente de un sistema dinámico adaptativo como la economía. Por lo tanto los organismos de política económica no pueden desconocer los valiosos enfoques de otras escuelas como es el caso del enfoque Post-Keynesiano. En este orden de ideas los modelos SFC aparecen como una herramienta adicional con muchos escenarios por explorar para que los hacedores de política tengan un panorama más amplio en las formulaciones y análisis de política económica en países donde la corriente principal ha sido el pensamiento único.

\section{MODELOS DE EQUILIBRIO GENERAL COMPUTABLE (EGC)}

\section{Una visión general de los Modelos de Equilibrio General Computable}

Esta metodología para la evaluación de la política económica tiene antecedentes los análisis económicos utilizando las matrices insumo-producto, los cuales consideran todas las transacciones realizadas entre los distintos sectores económicos reales de una economía, y donde los bienes producidos eran consumidos de manera completa por otro sector (Leontief, 1937). Trabajos posteriores significaron los inicios en adoptar modelos insumo-producto multisectoriales para la comprensión del funcionamiento de la economía e identificar algunos resultados de políticas. De ésta manera, emergieron avances considerables con el modelo construido para Noruega (Johansen, 1960), el cual se caracterizaba por introducir ecuaciones de comportamiento para consumidores y productores, asumiendo pleno empleo de recursos y encontrando el equilibrio económico a través de ecuaciones de balance macroeconómico sin la necesidad de que la economía se encontrase en un equilibrio óptimo. Este tipo de metodología a là Johansen fue aplicada posteriormente para varios países en particular aquellos en desarrollo ${ }^{1}$, y por otro lado, se desarrollaron trabajos relacionados con el equilibrio neo-clásico Walrasiano para hacerlo empíricamente útil para el análisis de políticas y proveer "un método general para la solución numérica explicita del modelo neoclásico" (Scarf y Hansen, 1973, pág 1).

Estos desarrollos se conocieron como Modelos de Equilibrio General Aplicado (MEGA) por su especificación ArrowDebreu y diferían de aquellos a là Johansen. A partir de 1985, los modelos MEGA empezaron a ser resueltos y calibrados con datos de cuentas nacionales adoptando de cierta manera el enfoque de Johansen e implementaron ecuaciones macroeconómicas de balance. A partir de allí los modelos de EGC y EGA empezaron a ser utilizados y resueltos de manera similar pero con algunas diferencias de presentación y de cierre del modelo(MitraKahn, 2008). En éste sentido, y siguiendo la tradición neoclásica (mainstream) de esta metodología ${ }^{2}$, se podría afirmar

\footnotetext{
1 Algunos ejemplos para países en desarrollo se pueden ver para Chile en Taylor y Black (1974), Brasil en Taylor y Lysy (1979), Turquia en Dervis (1975), Bangladesh en Ahmed (1974) y Corea del Sur en Adelman y Robinson (1977).

2 Para una discusión detallada de equilibrio general: Arrow y Hahn (1971) y Shoven y Walley (1992).
} 
que los consumidores cuentan con dotaciones iniciales de trabajo, capital y recursos, además de contar con un set de preferencias que determinan funciones de demanda para cada bien o producto. La demanda del mercado es la suma de la demanda de los consumidores y de la demanda de bienes intermedios.

Generalmente, los consumidores son agrupados de manera típica en un "agente representativo" con una demanda agregada y dotaciones totales. Sin embargo, los consumidores también pueden ser desagregados dependiendo de las respuestas que pretenda responder el modelo: es posible desagregar a la población por deciles de ingreso o por tipo de calificación. La demanda de los bienes en los mercados dependen en los precios y satisface la ley de Walras: en determinado set de precios, el valor to- tal de los gastos del consumidor es igual a los ingresos del consumidor. La tecnología generalmente es descrita por funciones de producción con rendimientos constantes de escala, y los productores maximizan ganancias. Si se considera la homogeneidad lineal de las ganancias en los precios y la homogeneidad de las funciones de demanda significa que solo los precios relativos tienen significancia en el modelo y el nivel de precios absoluto no tiene impacto en los resultados de equilibrio (Rutherford y Light, 2002). Metodológicamente, estos modelos son específicos para cada país, ya que requieren de una Matriz de Contabilidad Social que muestre los flujos de transacciones entre los distintos agentes económicos de una economía en particular. A continuación se muestra la estructura de una Matriz de Contabilidad Social (MCS) esquemática:

Figura 1: Estructura de la Matriz de Contabilidad Social (MCS)

\begin{tabular}{|c|c|c|c|c|c|c|c|c|c|}
\hline & Actividades & Productos & Factores & Hogares & Gobierno & RoW & Impuestos & $\begin{array}{l}\text { Ahorro- } \\
\text { Inversión }\end{array}$ & Total \\
\hline Actividades & & $\begin{array}{l}\text { Producción } \\
\text { doméstica }\end{array}$ & & & & & & & $\begin{array}{l}\text { Ingreso } \\
\text { Total }\end{array}$ \\
\hline Productos & $\begin{array}{l}\text { Consumo } \\
\text { intermedio }\end{array}$ & & & $\begin{array}{c}\text { Consumo } \\
\text { Hogares }\end{array}$ & $\begin{array}{l}\text { Consumo } \\
\text { Gobierno }\end{array}$ & $\begin{array}{c}\text { Exportacio- } \\
\text { nes }\end{array}$ & & Inversión & $\begin{array}{l}\text { Demanda } \\
\text { productos }\end{array}$ \\
\hline Factores & $\begin{array}{c}\text { Valor } \\
\text { Agregado }\end{array}$ & & & & & $\begin{array}{c}\text { Remunera- } \\
\text { ción factorial }\end{array}$ & & & $\begin{array}{l}\text { Ingreso } \\
\text { factores }\end{array}$ \\
\hline Hogares & & & $\begin{array}{c}\text { Remu- } \\
\text { neración } \\
\text { factorial }\end{array}$ & & Transf. & Transf. & & & $\begin{array}{l}\text { Ingreso } \\
\text { hogares }\end{array}$ \\
\hline Gobierno & & & & Transf. & & Transf. & $\begin{array}{l}\text { Recaudo } \\
\text { tributos }\end{array}$ & & $\begin{array}{l}\text { Ingreso } \\
\text { Gobierno }\end{array}$ \\
\hline RoW & & $\begin{array}{l}\text { Importacio- } \\
\text { nes }\end{array}$ & $\begin{array}{c}\text { Remu- } \\
\text { neración } \\
\text { factorial }\end{array}$ & Transf. & Transf. & & & & $\begin{array}{l}\text { Salida } \\
\text { divisas }\end{array}$ \\
\hline Impuestos & $\begin{array}{l}\text { Impuestos } \\
\text { Indirectos }\end{array}$ & $\begin{array}{l}\text { Impuestos } \\
\text { Indirectos }\end{array}$ & $\begin{array}{c}\text { Impuestos } \\
\text { directos }\end{array}$ & $\begin{array}{l}\text { Impuestos } \\
\text { directos }\end{array}$ & & & & & $\begin{array}{l}\text { Recaudo } \\
\text { tributario }\end{array}$ \\
\hline $\begin{array}{l}\text { Ahorro- } \\
\text { Inversión }\end{array}$ & & & & $\begin{array}{l}\text { Ahorro } \\
\text { Hogares }\end{array}$ & $\begin{array}{c}\text { Ahorro } \\
\text { Gobierno }\end{array}$ & $\begin{array}{l}\text { Ahorro } \\
\text { externo }\end{array}$ & & & $\begin{array}{c}\text { Ahorro } \\
\text { total }\end{array}$ \\
\hline Total & Costo Total & $\begin{array}{l}\text { Oferta de } \\
\text { Productos }\end{array}$ & $\begin{array}{l}\text { Gasto } \\
\text { factores }\end{array}$ & $\begin{array}{l}\text { Gasto } \\
\text { Hogares }\end{array}$ & $\begin{array}{c}\text { Gasto } \\
\text { Gobierno }\end{array}$ & $\begin{array}{l}\text { Entrada } \\
\text { divisas }\end{array}$ & $\begin{array}{l}\text { Recaudo } \\
\text { tributos }\end{array}$ & & \\
\hline
\end{tabular}


El equilibrio en este tipo de modelos se caracteriza por niveles de producción en cada sector y un determinado set de precios de tal manera que la demanda de mercado sea igual a la oferta de todos los bienes y productos. El nivel de desagregación tanto como sectores productivos como de agentes económicos es una decisión del modelador en función de las interrogantes que se pretenden responder a partir del modelo. Por tal motivo, un modelo que sea construido para analizar los efectos de la apertura comercial no sería adecuado para analizar los efectos y cambios en la política fiscal.

Un aspecto fundamental que debe destacarse, es que este tipo de modelos "mainstream" están diseñados para trabajar solamente con variables reales (teniendo en cuenta su origen walrasiano) y por lo tanto consideran neutral el dinero en el análisis. Este constituye como una de las diferencias fundamentales con el enfoque post-keynesiano, el cual considera la no-neutralidad del dinero como factor esencial para la comprensión del funcionamiento de los mercados financieros y de las economías modernas.

\section{Evaluación de la Política Económica en Colombia a partir de los Modelos EGC}

Los modelos de equilibrio general computable se han implementado de manera paulatina desde los años setenta en el país para el estudio y evaluación de la política económica ${ }^{3}$. De hecho, estos modelos han tenido amplia utilización en el país como el principal instrumento para la comprensión de las decisiones de política adoptadas en temas trascendentales para la economía nacional: apertura económica,

3 López et al (1994) presenta una revisión detallada de los modelos de simulación macroeconómica utilizando Modelos EGC en Colombia. planes de desarrollo, mercado laboral, política fiscal, entre otros. A pesar que a nivel internacional existen distintostipos de modelos de equilibrio general computable dependiendo del cierre del modelo 4 : Neoclásico, Keynesiano, Kaldoriano y Johansen (Delpiazzo, 2011:50), los modelos realizados en Colombia se han caracterizado por su orientación neoclásica. Este último aspecto puede ser atribuido al poco debate y reflexión existente en la literatura sobre las limitaciones de los desarrollos realizados por parte los de los diseñadores de los modelos. Considerando que su utilización fue extensiva en la década de los años 90 , los modelos construidos en las décadas previas tuvieron mayor influencia, determinando la importancia de la metodología para la evaluación de la política pública nacional ${ }^{5}$. De ésta manera se destacan trabajos como los de DeMelo y Robinson (1980) y Mayer (1983) que enfocaron su análisis en el comercio internacional y los efectos de cambios en la política comercial (aranceles y subsidios) sobre la distribución del ingreso y diversificación de exportaciones. Posteriormente, se presentaron desarrollos relevantes y originales como los de Londoño (1985) y Lora y Ocampo (1986) asociados a una contribución de perspectivas heterodoxas que sirvieron para analizar la distribución del ingreso. Finalmente, en a

4 En el cierre Neoclásico la inversión es endógena y determinada por el nivel de ahorro. En el cierre Keynesiano se permite desempleo, por tanto la oferta laboral es endógena. El cierre de Johansen es un punto intermedio: mantiene las consideraciones neoclásicas de la oferta pero también considera un nivel de inversión exógena. En el caso del cierre Kaldoriano (o Neo-Keynesiano) se considera un mecanismo de distribución del ingreso.

5 A mitad de la década de los años 1970, se creó el primer modelo EGC para Colombia y uno de los primeros prototipos de la región denominado SERES (Karl, 2004: 466), siendo sus principales los 3 módulos interrelacionados resueltos secuencialmente (demográfico, producción y consumo, educación y salud), lo que permitía analizar los efectos de la política económica en la salud, educación y en la estructura de la población. 
finales de los años ochenta se introduce el modelo "MEGA"6, el cual dio paso al modelo llamado DNP-COLCGE”.

Londoño (1990) introduce un modelo que se caracteriza por contar con un módulo "dinámico" que permitía analizar los cambios estructurales en la economía, analizando además efectos particulares sobre la distribución del ingreso y simulando cambios en políticas alternativas respecto al cambio tecnológico, acumulación de capital, y movilidad de factores (capital y trabajo). Eduardo Lora construye un modelo que combina la parte "real" de la economía con un sub-modulo financiero. Prácticamente, el sector real de la economía se basa en el trabajo de Londoño (1985) y Lora (1989a). El modulo financiero describe solamente los usos y fuentes de financiamiento de acuerdo con el equilibrio del sector real, representando una contraparte financiera a las transacciones de la economía real. Lora y Ramírez (1991) extienden su análisis en políticas monetarias y financieras, y sus efectos en los sectores agrícola, de la construcción, y urbanos.

En ambos trabajos, la inversión nominal es determinada por el ahorro disponible. A pesar de los importantes desarrollos durante los años 80s respecto a la utilización de modelos EGC en Colombia, la gran mayoría de estos se caracterizaron por ser modelos reales-estáticos. El primer intento de construir un modelo dinámico fue realizado por Zuleta y Arango (1994) para analizar los efectos de un boom petrolero (en precios). De hecho, utilizaron el modelo COLCGE, diferenciando sectores

\footnotetext{
6 Se incluyeron estructuras flexibles que permitieron capturar los efectos de cambios de política en el corto y largo plazo (Lora, 1989a y 1989b; Concha y Elorza, 1990).

7 Este modelo denominado DNP-COLCGE se utilizó para evaluar el Plan de Desarrollo "La Revolución Pacífica" de la administración Gaviria (DNP, 1991).
}

económicos en transables, no transables y petróleo. El modelo considera que los agentes tienen una previsión perfecta del futuro y maximiza el valor presente tanto de consumo como del valor de las empresas. La inversión estaba determinada por la q de Tobin y las rentas petroleras fueron consideradas como exógenas. Las predicciones del modelo estuvieron en línea con la literatura de la denominada "enfermedad holandesa", donde el sector transable es afectado después de una bonanza de precios del petróleo por una apreciación de la tasa de cambio real inducida por un incremento de flujos de capital en moneda extranjera.

Fedesarrollo construyó un modelo (Lora y Ramírez, 1990) que sirvió como base para analizar los efectos de las reformas estructurales y choques exógenos en la distribución del ingreso. Una extensión se enfocó en analizar los efectos de cambios en la política fiscal (Lora y Herrera, 1994). En un trabajo conjunto con Fedesarrollo, Bussolo, et al. (1998a y 1998b) construyó el modelo COGEM (Colombian General Equilibrium Model) para el análisis del mercado laboral. Se incluyó segmentación en el mercado laboral y legislación del salario mínimo, que a través de distintos cierres del modelo verificaron la creciente desigualdad salarial entre trabajadores calificados y no calificados. A inicios de la década de los años 2000, asesores externos contratados por el DNP desarrollaron un modelo para la evaluación de políticas fiscales denominado MEGATAX (Rutherford y Light, 2002). Este incluyó algunas especificaciones del mercado laboral que permitían ajuste de precios y migración entre trabajadores formales e informales a là Harris-Todaro. Una extensión de éste modelo fue la introducción de dinámica al mismo (Rutherford, Light, y Hernández, 2002) bajo formación de expectati- 
vas, manteniendo el funcionamiento del mercado laboral previo, lo que permitió al modelo de considerar la evaluación de efectos transicionales de una reforma fiscal. Por otro lado, se calibró un modelo multi-región utilizando la base de datos GTAP (Global Trade Analysis Project) con la finalidad de evaluar el impacto de acuerdos comerciales de libre comercio y sus efectos en la economía y bienestar (Light y Rutherford, 2003). Se consideraron los principales socios comerciales (USA, UE, y miembros de la CAN) y los resultados sugirieron beneficios positivos (pero pequeños) de esos acuerdos, principalmente porque Colombia ya gozaba de acceso preferencial con sus principales socios comerciales. Sin embargo, el modelo no capturó mejoras en la productividad como consecuencia de una mayor integración económica internacional.

Una de las recientes introducciones en la construcción de modelos EGC fue la integración con técnicas microeconométricas para mejorar los resultados de las simulaciones del modelo. Esto permitió evaluar la distribución del ingreso y cambios en la pobreza ocurridos con el proceso de liberalización de la economía durante la década del 1990 (Bussolo y Lay, 2003), sugiriendo que la reducción en aranceles contribuyó de manera significativa a la reducción de la pobreza entre 1988 y 1995, en particular en el sector rural. Con relación a la comprensión de los efectos de la crisis de 1999 y del incremento de las exportaciones sobre el empleo, la desigualdad y la pobreza, la Universidad de los Andes construye un modelo real para el corto plazo que asume pleno empleo de la fuerza laboral (Sánchez y Hernández, 2004) y sus resultados sugieren que en Colombia las políticas de liberalización comercial (reducción de aranceles) favorecen el crecimiento económico sin efectos negativos en la distribución del ingreso.
Una extensión del modelo MEGATAX utilizando técnicas microeconométricas (Karl, 2004) se enfoca en analizar los efectos de diferentes políticas fiscales sobre la pobreza ${ }^{8}$. Sus resultados sugieren que a pesar de que una reducción de aranceles aumenta el bienestar para todos los segmentos de la población, estos son contrarrestados por el ajuste de la posición fiscal del gobierno a través de instrumentos fiscales generando pérdidas en el bienestar. La inclusión de un IVA general podría afectar de manera negativa a varios grupos de la población, en particular si se consideran impuestos sobre productos de primera necesidad. Sin embargo, el modelo no considera aspectos importantes como desempleo, salarios mínimos u otras características de la economía. El Modelo (real) de Equilibrio General Computable de Fedesarrollo (MEGF) es utilizado para realizar un análisis de los impactos socioeconómicos y fiscales de la minería en Colombia (Fedesarrollo, 2008). Del mismo modo, y haciendo ajustes a su modelo MEGF se analiza el impacto económico de un tratado de libre comercio entre Colombia y la República de Corea del Sur utilizando un cierre macroeconómico a là Johansen?.

Durante 2008, con la intención de estimular la aplicación de la metodología y la discusión, se presenta un modelo estándar de equilibrio general computable para Colombia (Perdomo, 2008), el cual se caracteriza por ser estático-real y donde todas las decisiones que toman los agentes son opti-

8 Reducción de aranceles, introducción de un impuesto al valor agregado (IVA) general para todos los productos, reducciones en los flujos de capital e incrementos en las obligaciones del gobierno con el resto del mundo.

9 Se caracteriza por un cierre macroeconómico que considera, por un lado los supuestos neoclásicos del lado de la oferta y, por otro, establece un nivel exógeno de inversiones donde el mecanismo fundamental de ajuste ocurre a través de instrumentos de política fiscal endógena. 
mizadoras en un contexto de competencia perfecta. Sin embargo, éste es lo suficientemente flexible para realizar los cambios a nivel macroeconómico que considere pertinente el modelador y de esta manera introducir supuestos más elaborados que permitan alejarse del comportamiento optimizador. Finalmente, Núñez y González (2011) presentan los resultados para Colombia de la aplicación del modelo de equilibrio general computable desarrollado con el apoyo de UNDESA-CEPAL, denominado MACEPES-DNP (Modelo de Análisis de Choques Exógenos y de Protección Económica y Social), el cual se caracteriza por ser un modelo real dinámico-recursivo y con cierre macroeconómico neoclásico.

Considerando los modelos EGC desarrollados para Colombia, se evidencia que estos han sido construidos alrededor de las principales instituciones de investigación económica del país (DNP, Fedesarrollo, U. de los Andes) los cuales han abordado temas de importante trascendencia de la política económica nacional. Por consiguiente, varios de los modelos construidos guardan estrecha relación unos a otros o son extensiones de otros. La orientación teórica de las instituciones, y la evolución de los lenguajes de programación utilizados en la simulación parecen haber influido en éste sentido.

A pesar de que en el ámbito nacional, la construcción de este tipo de modelos ha presentado importantes avances desde sus inicios, en su mayoría se han caracterizado por ser modelos de sectores reales, esto debido a su orientación teórica neoclásica. No obstante los desarrollos realizados por la corriente neo-estructuralista permitieron incorporar el análisis del sector financiero a través de la construcción de la denominada Matriz de Contabilidad Social-Financiera y la Matriz de Flujos de
Transacción (Taylor, 1990). Sin embargo, es de resaltar que los modelos desarrollados han presentado importantes evoluciones, hasta integrar técnicas de simulación microeconométricas. Desafortunadamente, estas innovaciones tampoco permiten analizar el comportamiento del sector financiero, el cual cada día se convierte en un sector fundamental no solo en las economías más desarrolladas sino en economías emergentes como la colombiana. Se requieren modelos que contengan la parte financiera de la economía con la finalidad de establecer los costos y beneficios de las políticas de estabilización implementadas para reducir la inflación o aquellas para corregir los déficits fiscales y externos.

Es importante señalar que algunos supuestos en los modelos construidos, en particular aquellos que pretenden responder los efectos en la distribución del ingreso de determinado choque, consideran el pleno empleo de todos los factores primarios de producción, aspecto determinante cuando se analiza pobreza y desigualdad. De hecho, los supuestos de comportamiento y competencia significan que los factores de producción reciben todo el valor que producen, por tanto, asumir que todos trabajan y reciben la misma retribución limita la comprensión fundamental del por qué el estado de vulnerabilidad determinado por el desempleo y pago desigual.

Los modelos EGC se caracterizan por su flexibilidad para introducir distintos supuestos teóricos, y han logrado además extender su análisis al comportamiento del sector financiero, tal como lo demuestra la evidencia internacional ${ }^{10}$, pero la tipología

10 Trabajos desarrollados para países en desarrollo: Dervis, deMelo, y Robinson (1982); Bell y Srinivansan (1984); Taylor, Sarkar, y Rattso (1984); Easterly (1990); Rosensweig y Taylor (1990); Bourguignon, Branson, y deMelo (1989); Jemio (2001); Lay, Thiele, y Wiebelt (2004); Kahn (2004); Schweickert, Thiele y Wiebelt 
de los modelos EGC para Colombia se caracteriza por ser en su mayoría modelos estáticos reales con algunas excepciones de modelos dinámicos. Los desarrollos de modelos reales-financieros han sido nulos en el periodo reciente. Lo anterior puede ser debido, a las aplicaciones de los modelos construidos: en su mayoría se enfocan a analizar la política comercial y política fiscal, y sus efectos en la pobreza y distribución del ingreso. Esto de alguna manera ha limitado el desarrollo de modelos que incluyan el sector financiero de la economía como fundamental para comprender los choques tanto exógenos como de cambios de políticas tanto a nivel sectorial como a los distintos grupos de la población.

\section{MODELOS DE EQUILIBRIO GENERAL DINÁMICOS ESTOCÁSTICOS (DSGE) EN COLOMBIA}

Siguiendo los últimos desarrollos de la macroeconomía moderna ortodoxa, a finales de la década de los 90s comenzaron a utilizarse en entidades públicas de Colombia un tipo particular de modelos de equilibrio general caracterizados por el uso de un conjunto básico de supuestos derivados de los "nuevos clásicos" y los "nuevos keynesianos": individualismo metodológico, agentes optimizadores en un contexto intertemporal, agente representativo, expectativas racionales, rigideces nominales y reales, la existencia de múltiples choques estocásticos que afectan la economía, entre otros. El origen de la literatura moderna de modelos DSGE se remonta al trabajo de Kydland y Prescott (1982) sobre modelos de ciclos reales de los negocios (RBC), marco analítico que ha ido sufriendo

(2005); Yeldan, Voyvoda, y Telli (2008). Para modelos neoclasicos de Equilibrio General Aplicado (EGA) a economías en desarrollo consultar Shoven y Whalley (1984). múltiples extensiones, llegando a contar actualmente con un gran nivel de complejidad. En virtud de esto, en esta sección se van a presentar apenas las características más relevantes de los principales modelos utilizados en Colombia ${ }^{11}$, sin llegar a adentrarse en las especificidades matemáticas y estadísticas, pero enfatizando el objetivo de los modelos, las características particulares, las estrategias de estimación y las conclusiones de política.

El trabajo de Hamman y Riascos (1998) es una de las primeras aplicaciones de la literatura de modelos DSGE en Colombia y surgió de la necesidad de contar con una base teórica para la comprensión de los movimientos en las diferentes series macroeconómicas que se observan a lo largo del ciclo de negocios en Colombia. El modelo se centra exclusivamente en el sector real, el trabajo es indivisible (à la Hansen), existe un acceso imperfecto al mercado de capitales internacional, entre otros. Para simular el ciclo económico se considera que la economía está sujeta a cuatro choques estocásticos: (i) productividad (PTF), (ii) tasa de interés mundial real, (iii) trasferencias reales internacionales y (iv) gasto del gobierno. Los parámetros del modelo fueron calibrados para replicar el promedio histórico de algunas variables en Colombia (participación del consumo, inversión en el producto, entre otras) y siguiendo los valores utilizados en otros estudios.

11 La literatura de DSGE en Colombia se ha ido extendiendo rápidamente en los últimos años. Acá nos centraremos en los trabajos principales, intentando proporcionar una perspectiva histórica de la evolución de los mismos. Además, dadas las características más novedosas de los modelos SFC, enfatizaremos el uso de los modelos DSGE en política monetaria y en el análisis del sector financiero. El lector interesado en adentrarse más en la literatura puede consultar el trabajo de Revilla (2011) y la serie de trabajos que utilizan DSGE que puede encontrarse en la revista Borradores de Economía del Banco de la República. 
Para evaluar empíricamente el modelo se llevaron a cabo una serie de simulaciones y posteriormente se compararon los momentos (varianzas y autocorrelaciones) teóricos con los empíricos. Los autores destacan, entre otras cosas, la posibilidad de replicar teóricamente la alta volatilidad de la balanza comercial y su baja prociclicidad, y también reconocen la imposibilidad de reproducir la volatilidad del empleo.

A diferencia del anterior, el trabajo de Arias (2001) se enfoca en utilizar los modelos dinámicos estocásticos para comprender un suceso histórico particular, la crisis de finales del siglo pasado en Colombia. La hipótesis de trabajo que maneja Arias (2001) es que la nueva regulación bancaria implementada a finales del año 1998 generó un incremento en los costos de intermediación y una reducción en la versatilidad de los bancos, desembocando en una reducción de la productividad de los mismos, y un recrudecimiento de la crisis en Colombia. El marco teórico que maneja Arias (2001) son los modelos de acelerador financiero, en los cuales la intermediación financiera es tratada como una actividad sin costo, invisible e intangible. Se asume que los bancos operan bajo una tecnología de intermediación costosa, razón por la cual la tasa de interés de los préstamos es mayor que la de los depósitos. Las implicaciones que esto puede llegar a tener sobre la economía se obtienen del hecho que la producción se modela por medio de una función de producción cuyos insumos son el capital (fondos internos), los préstamos de los bancos (fondos externos) y la tierra (funciona como colateral). Con base en esto, Arias (2001) decide modelar la implementación de la nueva regulación bancaria como un choque negativo sobre la productividad de los bancos, lo que tiene por consecuencia un incremento en los costos de intermediación y, vía una reducción de los fondos externos y del precio de la tierra (reduciendo así el valor del colateral), un impacto negativo sobre el producto. Es así como se concluye en el trabajo que "la economía se tarda más en converger al estado estacionario en comparación con un marco sin fricciones financieras".

Los anteriores trabajos pueden considerarse como aproximaciones independientes al uso de modelos DSGE en Colombia. Desde comienzos del siglo XXI el Banco de la República viene trabajando en la construcción de un gran modelo DSGE para Colombia, con la intención de proporcionar una estructura teórica y empírica más sólida para la formulación de la política monetaria. El Banco de la República cuenta actualmente con el modelo PATACON (Policy Analysis Tool Applied to Colombian Needs), el cual es objeto de continuas mejoras. En González et al. (2011) se proporciona una descripción detallada de la estructura del modelo, el cual cuenta con una gran variedad de choques estocásticos (más de diez) y una estructura muy rica en términos de rigideces reales y nominales. Adicionalmente, este modelo se valida empíricamente haciendo uso de las últimas herramientas estadísticas, en particular por medio de estadística bayesiana. De este proyecto de investigación se han derivado una serie de trabajos que buscan analizar diferentes problemas económicos por medio de modelos DSGE.

A continuación se presenta un trabajo en particular como lo es de López et al. (2008), en el cual se tiene una modelación explícita y más detallada del sector financiero. López et al. (2008) introduce un sistema financiero simple en un modelo nuevo keynesiano de economía abierta. Se considera a los bancos comerciales como 
intermediadores en el mercado de fondos prestables: reciben los depósitos de los hogares (quienes los necesitan por la existencia de costos de transacción) y los prestan a las firmas (las cuales requieren de fondos externos para pagar los salarios por adelantado). Se asume que los bancos son firmas financieras especializadas en la producción de servicios financieros, por lo que cuentan con una función de producción que evidencia el costo inherente a la actividad de intermediación. El sistema financiero funciona además como puente entre las autoridades monetarias (banco central) y el sector privado, en la medida en que la política monetaria afecta directamente a los bancos comerciales y por intermedio de estos indirectamente al resto de la economía. Por un lado, los bancos deben mantener una proporción fija de reservas (especificada exógenamente por las autoridades monetarias) y pueden solicitar préstamos al banco central a una tasa de intermediación dada para cubrir sus necesidades.

Adicionalmente, los bancos comerciales también pueden solicitar préstamos en el exterior para financiarse. De acuerdo a esta estructura, el objetivo último del trabajo es evaluar el papel de la intermediación financiera costosa en la economía y que implicaciones tiene este papel sobre los mecanismos de transmisión de la política monetaria y la efectividad de la misma. Esto se evalúa por medio de funciones impulso respuesta que se obtienen de la consideración de cuatro choques estocásticos independientes: (i) a la tasa de interés repo, (ii) los requerimientos de reservas y (iii) un choque puro de estrés financiero. El autor también analiza dos escenarios posibles, uno en donde la intermediación financiera es costosa y el otro donde no se incurre en costos. Respecto al primer choque estocástico se obtienen los resultados tradicionales, en donde un aumento en la tasa repo desemboca en última instancia en una caída en la demanda agregada y una reducción en la inflación. Estos resultados son independientes del escenario considerado. En relación con los requerimientos de reservas, el autor encuentra que los resultados no son unívocos, pues el impacto sobre la economía depende de la estructura financiera (existencia o no de costos) y de la reacción de la tasa de interés repo (vía una regla de Taylor), y por lo tanto concluye que la política de requerimientos de reservas parece ser inefectiva y poco confiable. Por último, en relación el choque de estrés financiero, éste es modelado como una caída en el factor de eficiencia de los bancos, que tiene por resultado una reducción en la demanda agregada y en el producto, pero un incremento en la inflación en la medida en que se afecta negativamente la oferta del producto.

Los modelos DSGE siguen siendo usados de manera amplia en las diferentes instituciones del país a pesar que con la crisis de 2008 han recibido fuertes críticas por su incapacidad para predecir y comprender el debacle financiero de la década pasada. Es en este punto entonces donde la siguiente sección ahonda en modelos computacionales con otros enfoques como los modelos SFC (Stock Flow Consistent) que también podrían explorarse desde las instituciones que utilizan herramientas computacionales de simulación para el análisis de política económica en Colombia

\section{LOS MODELOS STOCK-FLOW CONSIS- TENT (SFC): UNA ALTERNATIVA DE LA TEORÍA POST-KEYNESIANA A LOS MO- DELOS ECONÓMICOS DOMINANTES.}

Una vez realizada una revisión sobre los principales modelos computables construidos para los análisis de política en 
el país se hace evidente que los modelos utilizados están estructurados alrededor de la teoría dominante y en las últimas décadas no existen intentos por construir o explorar modelos con otros presupuestos teóricos. Para el caso de los modelos de Equilibrio General Computable (ECG) si bien es cierto que se pueden construir con cierres de carácter más heterodoxo, los que se utilizan en Colombia tienen un espíritu fiel a la corriente principal. Adicionalmente los intentos recientes de construcción de modelos computacionales en el país siguen la tendencia de la ortodoxia global hacia el uso de modelos de equilibrio general dinámico estocástico (DSGE). Es entonces donde esta sección hace una invitación para explorar los modelos Post-Keynesianos (PK) SFC como una herramienta adicional que puede ser usada como instrumento de evaluación y recomendación de política públicas que enriquezcan las perspectivas de análisis en una visión más amplia y pluralista de la economía. Esta sección entonces presenta inicialmente algunos puntos teóricos de la teoría PK que son fundamentales para el planteamiento posterior de la estructura SFC y finalmente destaca algunos puntos relevantes de diferencia con los modelos tradicionales que hasta acá se exploraron.

\section{La teoría Post-Keynesiana y la importan- cia del crédito y la moneda endógena}

La economía Post-Keynesiana surge a partir de las contribuciones teóricas de reconocidos economistas como Joan Robinson, Nicholas Kaldor, Richard Kahn, Michal Kalecki, Abba Lerner y Hyman Minsky, para quienes en su visión las relaciones financieras influencian los procesos económicos tanto en el corto como en el largo plazo, debido a histéresis, path dependence e incertidumbre fundamental. De hecho, esta corriente incorporó una teoría monetaria de la producción (donde el dinero no se considera neutral) con un sistema financiero que genera no solamente créditos para actividades productivas sino también para otras actividades financieras, donde la tenencia de dinero y crédito influencia la velocidad, la oferta monetaria y por ende el producto en general.

En rol del crédito y del dinero en la escuela de pensamiento predominante ('mainstream') no es fundamental para el funcionamiento de la economía. En cambio, en los Post-Keynesianos, para que en una economía moderna se produzcan bienes y servicios, el rol del acceso al crédito es fundamental, lo cual podría definirse como una ley macroeconómica (Lavoie, 2014). Los Post-Keynesianos utilizan un enfoque teórico del sistema monetario que ha sido desarrollado por los banqueros durante siglos, en este enfoque, el dinero ingresa a la economía a través de la producción (y no solamente es medio de intercambio) y este es endógenamente determinado por la demanda de crédito bancario y sujeto a una convención social. La creación del dinero se comprende como resultado de la creación de nuevos pasivos dentro del proceso de expansión de ingreso que se considera un flujo más que un stock, el cual puede ser creado y destruido ${ }^{12}$. En éste tipo de economía, los prestamos crean depósitos debido a que el dinero es introducido en la economía a través de créditos iniciales otorgados a las empresas. Esta concepción descrita anteriormente se arropa en la denominada Teoría Endógena de la Moneda ${ }^{13}$ $\mathrm{y}$ es determinante tenerla presente en un enfoque de modelamiento computacional macroeconómico alternativo como los son

12 El circuito monetario de la producción o teoría monetaria de la producción, ha sido desarrollado en Francia (Parguez, 1980; Lavoie, 1984).

13 Para una presentación formal del modelo revisar Lavoie (2014). 
los modelos SFC (Stock Flow Consistent) que se presentan a continuación.

\section{Una introducción conceptual al modela- miento Stock-Flow Consistent (SFC)}

El enfoque Post-Keynesiano SFC tiene sus orígenes en los trabajos de Wynne Godley y Francis Cripps (1983), quienes inicialmente buscaron desarrollar modelos que mostraran de manera consistente la interrelación de los sectores real y monetario de la economía. No obstante vale la pena resaltar que los trabajos de estos dos autores tienen fuertes raíces en el trabajo pionero de Copeland (1949) en el cual se reconoce un enfoque de contabilidad social para los flujos de dinero y un aporte inicial a la integración de los flujos reales y financieros. Características como el sistema de entrada cuádruple, fundamental para los SFC es parte del legado de Copeland a esta metodología. Además no se puede dejar de hacer alusión al trabajo de Tobin y sus coautores (Backus et al, 1980) quienes ponen una semilla metodológica para el análisis stock flujo a pesar de que las ecuaciones de sus modelos manejan un corte más ortodoxo.

La metodología SFC parte de un enfoque de contabilidad donde cada transacción tiene una contraparte, es decir toda compra tiene una venta asociada y los saldos financieros de cada sector están ligados a un cambio en la variable stock correspondiente (Sistema de Cuádruple Entrada). Esta premisa cobra gran importancia cuando se tiene una economía moderna donde los créditos tienen un rol fundamental; en este sentido los flujos financieros que surgen en el proceso de producción e intercambio tienen que ser involucrados en los modelos que describen la economía (Toporowski y Michell, 2011).
En los primeros estándares iniciales de las cuentas nacionales no se le dio un espacio significativo a los bancos y a los intermediarios financieros. Sin embargo, el nuevo sistema de cuentas nacionales publicado en 1968 por la ONU despejó muchas dudas y planteó un esquema teórico capaz de lograr la integración entre las cuentas de ingresos nacionales y las transacciones financieras, los stock de capital y las hojas de balance. Este nuevo esquema de contabilidad, mucho más robusto e integrado, fue validado con la revisión de 1993 y despejó las dudas existentes en los primeros planteamientos. Sin embargo "el problema hoy por hoy ya no es la ausencia de datos apropiados, sino la falta de voluntad de muchos de los macroeconomistas del mainstream para incoroporar los flujos financieros y los stocks de capital en sus modelos, obsesionados como ellos son con el agente representativo microeconómico optimizador" (Godley y Lavoie, 2007, p. 25).

El enfoque Stock flujo consistente (SFC) se encuentra basado en cinco aspectos determinantes, algunos de ellos revindicados por Tobin en su discurso de recepción del Nobel en 1982 según afirman Godin y Caverzaci (2013), estos aspectos son: Precisión respecto al tiempo, seguimiento de los stocks, varios tipos de activos y tasas de retorno, modelación financiera y de las operaciones de política monetaria y presupuestos sectoriales. En los modelos SFC es de vital importancia señalar que las relaciones entre stocks y flujos evolucionan a través del tiempo; en otras palabras pueden ser identificados como "modelos de corte evolutivo los cuales describen una economía que se mueve hacia delante de forma no ergódica en el tiempo histórico" (Godley y Lavoie, 2007, p. 9). En estos modelos, no son necesarias las expectativas racionales dado que los agentes reaccio- 
nan a los desequilibrios vía funciones de ajuste parcial.

De hecho, en los modelos de Godley, los agentes económicos son razonablemente racionales de acuerdo con nueva información y normas. Estas normas están relacionadas por ejemplo con la función de consumo de las familias que en estos modeloses determinada por su stock realde capital acumulado en periodos anteriores junto con su flujo esperado de ingreso disponible en un periodo actual. En el caso de las empresas, operan dentro de un rango normal de producción en el cual el costo por unidad de producto es constante, y basan sus decisiones (producción, empleo, $\mathrm{y}$ precios) en la cantidad que esperan vender considerando sus inventarios. Para el gobierno, su decisión se concentra en la relación deuda pública-PIB. Además, el funcionamiento de los mercados presenta ajustes de cantidades (y no de precios como en el mainstream), donde la oferta es igual a la demanda en determinados periodos o por la presencia de buffers: inventarios y préstamos para las empresas, depósitos en dinero para las familias, emisión títulos de deuda para el gobierno, tenencia de títulos de deuda y avances del banco central para los bancos privados. Este tipo de modelos permiten identificar quienes son los dueños de la riqueza, su tamaño preciso y que hacen con ella. De manera simultánea, permiten identificar como el tamaño y la distribución de los stocks financieros cambian con el tiempo (Dos Santos, 2009).

\section{Entendiendo la Estructura de los modelos SFC}

La estructura de un modelo SFC reposa sobre dos aspectos fundamentales, el primero de ellos es la estructura contable que se presenta siempre en dos matrices, (i) la matriz de balance, que muestra los stocks financieros del modelo y (ii) la matriz de transacciones, que muestra los flujos y como elemento novedoso involucra los cambios en los stocks de activos y pasivos financieros. El segundo aspecto fundamental de los modelos SFC son las ecuaciones de comportamiento y las identidades del modelo, que en términos operativos permitirán el desarrollo y la solución(analítica o computacional) del mismo, con la ayuda de la estimación o calibración de los parámetros del modelo. A continuación se revisan cada uno de estos dos aspectos.

Respecto al primer aspecto que es el contable se tienen las dos matrices mencionadas anteriormente. La matriz de balance fundamentalmente describe los stocks de activos y pasivos de cada sector y su interrelación con otros sectores. Bajo esta lógica cada activo financiero de un sector debe tener una contraparte como pasivo financiero en uno o más sectores. Las matrices de balance pueden tener diversos ítems, como dinero (highpowered money), depósitos, bonos, acciones y títulos financieros. Siempre cada uno de ellos comportándose como activo de un sector(es) y pasivo de otro(s). Por otra parte, la matriz de transacciones describe en su primera parte las variables estándar de las cuentas nacionales como consumo $(C)$, gasto público $(G)$ y demás variables que se encuentran en los libros tradicionales. Sin embargo, adicionalmente este tipo de matrices describen los cambios en los stocks de activos y pasivos financieros descritos en la matriz de balance. Es posible construir una tercera matriz de apoyo que es la denominada matriz de comportamiento, esta se puede entender como una forma particular de representar la matriz de transacciones especificando el comportamiento de los sectores. Por ejemplo para variables como el Consumo 
(C) y la Inversión (I), es posible especificar el comportamiento de las variables para cada sector, así entonces para un casa en que se quiera especificar variables de oferta y de demanda se pueden adicionar subíndices (s, d) a las diferentes variables. Por ejemplo el "monto de consumo C, demandado por los hogares como $\mathrm{Cd}$ es igual al monto de consumo ofrecido por las firmas Cs. Entonces este monto va para pago de salarios, inversión en bienes de capital, bonos emitidos por bancos a las firmas y a los hogares respectivamente" (Kinsella, 2011, p.5). De esta forma se da un proceso de interacción entre los sectores.

Hechas estas precisiones entonces se puede dar paso al segundo aspecto fundamental en la construcción del modelo SFC, que como ya se dijo anteriormente está asociado a escribir identidades contables que garanticen la consistencia del modelo y a la vez un grupo de series de ecuaciones de comportamiento que especifican los comportamientos para cada sector. Así entonces para el sector de los hogares se puede especificar ecuaciones de ingreso disponible dadas por la diferencia entre salarios (flujo de ingreso) y otros flujos de egreso como Consumo y deuda. Estos flujos de egreso conformaran ecuaciones de otros sectores como las firmas y los bancos que tendrán sus respectivas ecuaciones para describir cambios en sus flujos y stocks. De esta manera se construye un sistema de $n$ ecuaciones con $n$ incógnitas. Es importante tener presente que muchas de las ecuaciones cuentan con parámetros que puede ser o estimados econométricamente o calibrados a partir de datos reales para un estado estable asociado a las matrices de balance y transacciones. Una vez entonces se logran tener los parámetros calibrados o estimados del modelo se puede proceder a desarrollar el modelo y su solución de estado estable para cada una de las variables.
Para modelos pequeños es posible realizar soluciones analíticas pero modelos grandes y con especificidades más realistas pueden ser muy grandes y necesitan del uso de métodos numéricos en el computador para encontrar la solución. Una vez se tiene una solución se procede a realizar experimentos modificando las variables de interés en diferentes escenarios que son comparados con el estado estable para analizar conclusiones relevantes para la economía. Algunas críticas a este modelos son relativas a la metodología que en muchas ocasiones es similar a la de otros métodos de la corriente principal, no obstante no se puede olvidar el trasfondo teórico y el espíritu heterodoxo (ecuaciones donde es explicito el dominio de la demanda, dinero endógeno, etc.) en las ecuaciones.

La descripción proporcionada acá es bastante superficial sin embargo el lector interesado en una aproximación más detallada a los modelos SFC puede revisar el texto de "Monetary Economics" (Godley y Lavoie, 2007) donde se explica paso a paso la metodología que se describió acá brevemente y se incluyen detalles de las simulaciones computacionales de esta estructura de análisis. Actualmente estos modelos tienen una amplia aceptación en la tradición Post-Keynesiana y se caracterizan por la interacción de todos los sectores de la economía con al menos otro sector, en otras palabras todo viene de algún lugar y va hacia otro, sin agujeros negros.

\section{Algunos puntos novedosos a resaltar de los modelos SFC respecto a las formas de modelamiento tradicionales}

Los modelos SFC por sus características antes mencionadas han probado ser una herramienta altamente útil por anticipar y seguir la evolución de la reciente recesión 
de la economía norteamericana. Este es el caso puntual del Modelo de Levy Institute para Estados Unidos (Levy Institute Model for USA -"LIMUSA") que es la evolución de los primeros modelos en los que Godley usaba el enfoque SFC para observar desbalances e inestabilidad creciente. La principal característica del modelo LIMUSA es que es el primero en ser capaz de realizar algunas predicciones de una manera muy sistemática puesto que este permite la comparación de diferentes escenarios resultantes de diferentes políticas. (Godin y Caverzaci, 2013). Actualmente existen trabajos con un enfoque similar para Grecia (Papadimitrou, Zezza y Nikiforos, 2012) que también se construyen desde Levy Institute y el trabajo más completo publicado para el Reino Unido por el Banco de Inglaterra en Burgess et al. (2016), además de algunos desarrollos para países emergentes como Colombia (Escobar, 2016).

Existen varias diferencias relevantes de los modelos SFC heterodoxos con los modelos computacionales ortodoxos neoclásicos y neo keynesianos, sin embargo uno de los puntos que vale mencionar de manera insistente es la integración de los flujos reales y financieros de la economía. Esta característica le permitió a Godley y su equipo del Levy Economics Institute anticiparse a la reciente crisis de la economía norteamericana en un marco formal (LIMUSA). Y es que en 2006 Godley y Zezza publicaron un artículo titulado "Debt and Lending: A Cri de Coeur" donde ellos demuestran la dependencia de la economía norteamericana del crecimiento de la deuda. Ellos argumentan allí que esto sumirá a Estados Unidos en una recesión antes de 2010 (Godley y Zezza, 2006). Este hecho hizo merecedor a Godley de una importante nota en el prestigioso diario The New York Times ${ }^{14}$ en Septiembre de 2013

14 Embracing Wynne Godley, an Economist Who Modeled the Crisis. By JONATHAN SCHLEFER. a pesar de su deceso en 2010. Este punto es altamente relevante puesto que los modelos de equilibrio general dinámicos estocásticos (DSGE) no tuvieron la capacidad de predecir y ni siquiera dar señales de alerta sobre la crisis que sacudió al corazón financiero hace un lustro. Y es que en los modelos DSGE el "producto potencial, determinado exclusivamente por factores de oferta, marca la senda de la economía y las posibles desviaciones entre el producto potencial y el real se corrigen con variaciones en precios y salarios, en la mejor línea Lucas-Romer" (Garrido, 2013, p.65). Es importante destacar que la interacción entre flujos reales y financieros en este tipo de modelos está acompañada por un reconocimiento claro y puntual del rol del dinero en el proceso productivo y por lo tanto hay una gran coherencia teórica al modelar el sector bancario y sus interacciones con los otros sectores.

Adicionalmente y siguiendo uno de los pivotes de la teoría Post-Keynesiana el dinero se asume como endógeno, lo cual implica que este viene determinado por las demandas de crédito y no por las acciones del banco central para fijar la cantidad de dinero en la economía. Otro de los puntos muy relevantes está asociado a la combinación del enfoque contable con principios claros de la teoría Post-Keynesiana. Y si bien es cierto que el enfoque de contabilidad en la modelación macro no es exclusivo de Godley $^{15}$, muchas de las características de sus modelos marcan una distancia teórica

Published: September 10, 2013. Disponible en :http://www.nytimes.com/2013/09/11/business/ economy/economistsembracing-ideas-of-wynnegodley-late-colleague-who-predicted-recession. html?pagewanted=all

15 Muchos modelos macro ortodoxos muestran un enfoque contable, como es el caso de los modelos ortodoxos de Tobin y su equipo de Yale, e incluso algunos modelos DSGE muestran consistencia StockFlujo. Sin embargo los cierres y la base teórica están en otra orilla conceptual. 
importante; y es que estos modelos son guiados por la demanda (demand-led) y consecuentemente aceptan el principio de la demanda efectiva sin suponer pleno empleo. Por otra parte se anclan en la idea de competencia imperfecta y responden a la teoría micro Postkeynesiana con una fijación de precios vía mark-up. Otra clara distinción es la exclusión del fuerte supuesto del agente maximizador y más bien se centra en un enfoque evolutivo de la economía que sigue normas stock-flujo; $\mathrm{y}$ es que muchos de los modelos macro de inspiración Neo Keynesiana se basan en modelos de expectativas racionales donde los agentes maximizan y logran el equilibrio.

A diferencia de esta noción los modelos SFC a là Godley-Lavoie se basan en una racionalidad procedimental con agentes que reaccionan a desequilibrios pasados relativos a normas. (Godley y Lavoie, 2007, p. 493). Siguiendo una amplia tradición heterodoxa estos modelos también reconocen instituciones y clases sociales al trabajar una estructura económica de diversos sectores interrelacionados junto con una alusión explicita a una dinámica del tiempo histórico donde se tiene en cuenta los stocks acumulados al final de un periodo e inicio del siguiente. Un supuesto importante es considerar el stock nominal del dinero endógenamente determinado donde los bancos centrales no tienen control sobre éste stock, como se mencionó anteriormente. El cambio en el stock de dinero es ocasionado por la demanda de créditos del sector privado por tanto, el sector bancario debe adaptar los depósitos bancarios para mantener las reservas privadas requeridas por el banco central. Este supuesto indica que las tasas de interés tienen que ser exógenas (al menos es cierto para la tasa de descuento del banco central), mientras que en el mainstream, la tasa de interés tiene que equilibrar la oferta y demanda de dinero Recientemente los modelos SFC, dada su amplia difusión entre la comunidad heterodoxa, han empezado a mostrar progresos e incluso avances en la microfundamentación para dar respuestas contundentes a la microfundamentación ortodoxa en los DSGE. Y es que la combinación de modelamiento basado en agentes (ABM) y el enfoque SFC puede abrir nuevas puertas para el avance teórico heterodoxo. Así entonces, Bezemer (2011) entre otros, creen firmemente en que la "combinación de la flexibilidad del modelamiento basado en agentes con las consistencia entre stocks y flujos de un sistema brinda un marco que provee $y$ asegura la compatibilidad entre variables reales y financieras" (Godin y Caverzaci, 2013, p.13).

La metodología SFC-ABM se puede interpretar como una respuesta de la heterodoxia a los dominantes DSGE. Simultáneamente se puede ver como una forma de presentar una microfundamentación alternativa a los modelos macro como respuesta a las críticas asociadas a falacias de composición. Más allá de esto, esta metodología permite con simples reglas procedimentales entender como los micro comportamientos generan tendencias macro complejas (Godin y Caverzaci, 2013, p.13). Estos modelos tienen un gran potencial, sin embargo aún hay un gran trabajo por hacer puesto que gran parte de los trabajos asociados a SFC permanecen en su dimensión teórica y pocos se arriesgan a trabajos empíricos, con contadas excepciones como el modelo Levy para Estados Unidos y el de Reino Unido que se mencionaron antes. No obstante, los modelos Stock-Flujo pueden retroalimentarse de las innovaciones en otras áreas de la economía como la economía del comportamiento y 
algunos avances en economía computacional.

Dado entonces este marco explicativo es claro que existen opciones alternativas serias para el modelamiento computacional con un trasfondo teórico riguroso. Este tipo de herramientas pueden añadirse a las ya existentes como instrumentos adicionales para el análisis de política pública como ya lo han empezado a hacer Bancos Centrales con políticas estándar que quieren involucrar estas herramientas, como el caso reciente del Banco de Inglaterra antes referenciado.

\section{CONCLUSIONES}

La crisis financiera global ha introducido retos no solo a los gobiernos sino a los economistas y los modelos mediante los cuales se dan sugerencias de política pública. Adicionalmente una mayor integración económica internacional ha demostrado la mayor vulnerabilidad de las naciones, no solo por el creciente intercambio de bienes y servicios sino por la mayor integración de los mercados financieros. En este orden de ideas los modelos CGE y los DSGE han sido herramientas muy influyentes en los Bancos Centrales y en las oficinas de planeación de muchas naciones y Colombia no ha sido la excepción como se mostró anteriormente. Sin embargo dichos modelos reposan bajo el mismo manto teórico de la corriente estándar en economía, donde los agentes optimizadores son predominantes y el sector financiero no tiene la relevancia que se le debería dar en una economía con un alto predominio de motivos y roles financieros. En este punto entonces vale la pena mirar alternativas a las formas de modelamiento más conocidas y utilizadas popularmente en los ámbitos técnicos de los organismos. La teoría Post Keynesiana presenta algunos elementos asociados a una teoría moderna del dinero donde se insiste en la endogeneidad de la moneda y la capacidad del sistema financiero para crear dinero bancario que posteriormente es destruido. En este enfoque se le da igual importancia al seguimiento de los flujos financieros y de los stocks para determinar la acumulación de riqueza de los agentes, y la capacidad de estos últimos para destinar sus ahorros en una variedad de activos financieros. Una potencial propuesta desde la teoría Post-Keynesiana está asociada a los modelos SFC (Stock Flow Consistent) que se describieron en la última sección del artículo.

Así entonces este artículo hace un llamado a explorar en el ámbito de la política económica nacional los modelos SFC, e incluso repensar y reanalizar muchos hechos históricos analizados anteriormente con modelos CGE y DSGE a la luz de la metodología Stock-Flujo. Es hora de empezar a dar unos primeros pasos para extender este tipo de metodologías alternativas a economías emergentes donde los encargados de diseñar y evaluar la política económica muchas veces simplemente replican las metodologías ortodoxas sin reflexionar sobre su pertinencia. Y este es el caso de Colombia donde como se ha mostrado existe una tradición importante de modelamiento macroeconómico basado en esquemas ortodoxos. De esta forma el Banco de la República y el DNP más allá de olvidar completamente el uso de metodologías tradicionales deberían asumir la tarea de explorar enfoques de modelamiento adicionales y alternativos siendo los SFC una buena posibilidad para tener un marco más amplio de herramientas para comprender la economía.

Para el caso de una economía como la colombiana, donde no se puede minimizar el rol del sistema financiero, es necesario 
contar con modelos no tradicionales que permitan comprender mejor el funcionamiento de la economía para así contribuir al debate en la formulación de la política pública, y no esperar a que la economía se encuentre en senderos no deseados de cambio estructural para contrastar programas de ajuste traumáticos provenientes de posiciones radicales ortodoxas. De esta manera la agenda de investigación nacional debe enfocarse hacia la construcción de un primer esquema de modelo empírico con las características antes mencionadas y que han mostrado interesantes resultados. Simultáneamente hoy en el mundo la agenda plantea líneas de investigación asociadas a la modelación SFC-ABM, la desagregación de activos del sistema financiero, dinámicas multisectoriales (a là (GE) y SFC teóricos de inestabilidad no lineales.

\section{REFERENCIAS BIBLIOGRÁFICAS}

Adelman, I., y Robinson, Sh., (1977), "Income distribution policies in developing countries: A case study of Korea", Stanford: Stanford University Press.

Ahmed, F. (1974), "Migration and employment in a multi-sector model: an application toBangladesh", unpublished $\mathrm{PhD}$ dissertation, Princeton University.

Arias F. (2001), "Banking productivity and economic fluctuations: Colombia 1998-2000", University of California, Los Angeles.

Arrow, K., y Hahn, F. (1971), “General competitive analysis”, San Francisco: Holden-Day.

Bell C., y Srinivasan N., (1984), "On the Uses and Abuses of Economywide Models in Development Planning Analysis", en M. Syrquin, L. Taylor and L. E. Westphal (eds.), Economic Structure and Performance. New York: Academic Press.

Backus, D., Brainard, W. C., Smith, G., y Tobin, J. (1980), "A model of US financial and nonfinancial economic behavior", Journal of Money, Credit and Banking, 259-293.

Bezemer (2011), "Causes of Financial Instability: Don't Forget Finance”, Working Paper No. 665, Levy Economics Institute.

Bourguignon, F., Branson, W., y deMelo, J. (1989), "Macroeconomic Adjustment and Income Distribution: A macro-micro simulation model”, OECD Development Centre.

Burgess, S., Burrows, O., Godin, A., Kinsella, S \& Millard, S. A Dynamic Model of Financial Balances for the United Kingdom (September 2, 2016). Bank of England Working Paper No. 614. Available at SSRN: https://ssrn.com/abstract=2835386 or http://dx.doi.org/10.2139/ssrn.2835386

Bussolo, M., y Roland-Holst, D. (1998a), “Colombia and the NAFTA”, Serie Documentos de Trabajo, No. 2, enero, Fedesarrollo.

Bussolo, M., Roland-Holst, D., y Van der Mensbrugghe, D. (1998b), "The Technical Specification of Fedesarrollo's Long Run General Equilibrium Model", Serie Documentos de Trabajo, Numero 4, marzo, Fedesarrollo.

Bussolo, M., y Lay, J. (2003), “Globalization and poverty changes in Colombia”, OECD. 
Cicowiez, M. (2011), "Un Modelo de Equilibrio General Computado para la Evaluación de Políticas Económicas en Argentina: Construcción y Aplicaciones", Tesis de Doctorado. Universidad Nacional de la Plata, Argentina.

Concha, A., y Elorza, J. (1990), "Impacto Macroeconómico del programa de Modernización de la Economía Colombiana: Aplicación de un modelo de Equilibrio General Computable", Revista de Planeación y Desarrollo, Vol. 22 No. 3-4.

Copeland, M. A. (1949), "Social accounting for money flows”, Accounting Review, 254-264.

Delpiazzo, E. (2011), "The Mozambican Participation in SADC: A Liberalization Process through different models and different closures", Doctoral Thesis, Universita Cattolica del Sacro Cuore.

DeMelo, J., y Robinson, Sh. (1980), "The impact of trade policies on income distribution in a planning model for Colombia”, Journal of Policy Modeling, Vol. 2, No. 1: 81-100.

Departamento Nacional de Planeación - DNP (1991), "La Revolución Pacífica”, Ed. Presencia.

Dervis, K., deMelo, J., y Robinson, Sh. (1982), “General Equilibrium Models for Development Policy”, Cambridge: Cambridge University Press.

Dervis, K. (1975), "Substitution, employment, and intertemporal equilibrium in a non-linear multisector planning model for Turkey", European Economic Review, Vol. 6:77-96.

DosSantos, C. (2009), "A primer on SFC Models. Presentation at Unicamp”, Campinas, Brasil.

Easterly, W. (1990), "Portfolio effects in a CGE model: Devaluation in a dollarized economy", en Taylor, L. (ed.), Socially Relevant Policy Analysis: Structuralist Computable General Equilibrium Models for the developing world, Cambridge, MA: MIT Press.

Escobar, A. (2016), Stock-Flow Consistent Models for Developing Countries: The case of Colombia". 19th Annual Conference on Global Economic Analysis, GTAP.

Fedesarrollo (2008), "La Minería en Colombia: Impacto Socioeconómico y Fiscal" Cuaderno No. 25, Bogotá.

Garrido, A. (2013), “El enfoque macroeconómico consistente de Wynne Godley una exposición”, Revista de Economía Crítica, Nº15, primer semestre, 61-72.

Godin, A., y Caverzaci, E (2013), "Stock-flow Consistent Modeling through the Ages", Working Paper Series No. 745. Annandale-on-Hudson, NY: Levy Economics Institute of Bard College.

Godley, W., y Cripps, F. (1983), “Macroeconomics”, London, Fontana.

Godley, W., y Lavoie, M. (2007), "Monetary Economics An Integrated Approach to Credit, Money, Income, Production and Wealth", New York: Palgrave MacMillan.

Godley, W., y Zezza, G. (2006), “Debt and lending: A Cri de Coeur”,(No. 06-4), Levy Economics Institute.

González, Mahadeva, Prada y Rodríguez (2011), "Policy Analysis Tool Applied to Colombian Needs: PATACON Model Description”, Borradores de Economía No. 656.

Hamman y Riascos (1998), "Ciclos económicos en una economía pequeña y abierta - Una aplicación para Colombia”, Borradores de Economía No. 89. 
Jemio, L. (2001), "Debt, Crisis, and reform in Bolivia: biting the bullet", International Finance and Development Series, Palgrave Macmillan.

Johansen, L. (1960) "A multi-sector study of economic growth", Amsterdam: North Holland Publishing Company.

Kahn, H. (2004), "Using macroeconomic computable general equilibrium models for assessing poverty impact of structural adjustment policies", ADB Institute Discussion Papers, No. 12.

Karl, C. (2004), "How can tax policies and macroeconomic shocks affect the poor? A Quantitative Assessment using a Computable General Equilibrium Framework for Colombia", Revista Ensayos sobre Política Económica ESPE, No. 46, 2: 450-519.

Kinsella, S. (2011), "Words to the Wise: Stock Flow Consistent Modeling of Financial Instability".

Kydland, F. E., y Prescott, E. C. (1982), "Time to build and aggregate fluctuations". Econometrica: Journal of the Econometric Society, 1345-1370.

Lavoie, M. (1984), "The endogenous flow of credit and the post Keynesian theory of money". Journal of Economic Issues, 18(3), 771-797.

Lavoie, M. (2014), "Post-Keynesian economics: new foundations". Edward Elgar Publishing.

Lay, J., Thiele, R., y Wiebelt, M. (2004), "Pro-poor growth in Bolivia: Accounting for external shocks and policy reforms", Kiel Working Paper Series No. 1231.

Leontief, W.W. (1937), "Inter-relation of prices, output, savings and investment", Review of Economics and Statistics, 19: 109-132.

Light, M. K., y Rutherford, T. F. (2003), "Free trade area of the Americas an impact assessment for Colombia", DNP.

Londoño, J. (1985), "El papel macroeconómico del Mercado de alimentos: un modelo de equilibrio general", Revista Coyuntura Económica, Fedesarrollo, Vol. 15 No. 4, diciembre.

Londoño, J. (1990), "Income distribution during the structural transformation, Colombia 1938-1968" PhD thesis, Harvard University.

López, E., Ripoll, M., y Cepeda, F. (1994), “Crónica de los Modelos de Equilibrio General en Colombia”, Borradores Semanales de Economía, Banco de la Republica.

López, Prada y Rodríguez (2008), "Financial Accelerator Mechanism in a Small Open Economy", Borradores de Economía No. 525.

Lora, E. (1989a), "Coffee and Oil shocks in the short and the long run: an application of alternative CGE models for Colombia", Mimeo, Fedesarrollo.

Lora, E. (1989b), "Real and Financial interactions in a Computable General Equilibrium Model for Colombia", Mimeo, Fedesarrollo.

Lora, E., y Herrera (1994), "Tax incidence in Colombia: A General Equilibrium Analysis", Fedesarrollo. Mimeo. Lora, E., y Ocampo, J.A. (1986), "Política Macroeconómica y distribución del Ingreso en Colombia: 1980-1990”, Coyuntura Económica Vol. 16, 3: Octubre. Biblioteca Virtual del Banco de la Republica. 
Lora, E., y Ocampo, J. A. (1986), "Política macroeconómica y distribución del ingreso en Colombia: 1980-1990", Coyuntura Económica, 16(3), 109-158.

Lora, E., y Ramírez, J. (1990), "Macroeconomía, distribución del ingreso y sector informal”, Fedesarrollo, Bogotá.

Lora, E., y Ramírez, J. (1991), "Ajuste estructural y desarrollo humano en Colombia: Una evaluación de las opciones".

Mayer, T. (1983), "Effects of export diversification in a primary commodity exporting country: Colombia", Journal of Policy Modeling, 5:233-252.

Mitra-Kahn, B. (2008), "Debunking the Myths of Computable General Equilibrium Models", SCEPA Working Paper 2008-1. The New School for Social Research.

Núñez, J., y González, N. (2011), “Colombia”, en: Sánchez, M. V., \& Sauma, F. (eds.), Vulnerabilidad económica externa, protección social y pobreza en América Latina. CEPAL.

Papadimitrou, D. B., G. Zezza, and M. Nikiforos (2013). “A LEVY Institute Model for Greece”, Technical Paper. Annadale-on-Hudson, NY: Levy Economics Institute.

Parguez, A. (1980), "Profit, épargne, investissement: Eléments pour une théorie monétaire du profit”.

Perdomo, Á. (2008), “Modelo estándar de equilibrio general computable”, Archivos de Economía DNP, Documento 342.

Revilla (2011), “Modelos de equilibrio dinámicos y estocásticos para Colombia 1995 - 2011”, Ecos de Economía, Universidad EAFIT.

Rosensweig, J., y Taylor, L. (1990), "Devaluation, Capital Flows, and Crowding-Out: a CGE model with Portfolio Choice for Thailand", en Taylor, L. (ed.), Socially Relevant Policy Analysis: Structuralist Computable General Equilibrium Models for the developing world, Cambridge, MA: MIT Press.

Rutherford, T., y Light, M. (2002), "A General Equilibrium model for tax policy analysis in Colombia: The MEGATAX model”, Documento No. 188, Mayo, Departamento Nacional de Planeación.

Rutherford, T. F., Light, M. K., y Hernández, G. A. (2002), “A dynamic general equilibrium model for tax policy analysis in Colombia", Departamento Nacional de Planeación.

Sánchez, F., y Hernández, G. (2004), "Colombia: Aumento de las exportaciones y sus efectos sobre el crecimiento, empleo y pobreza”, Desarrollo y Sociedad, No 53: 193-226, marzo.

Scarf, H.E., y Hansen, T., (1973), "The Computation of Economic Equilibria”, Cowles Foundation for Research in economics at Yale University, Monograph No. 24, New Haven, CT and London, UK: Yale University Press.

Schweickert, R., Thiele, R., y Wiebelt, M., (2005), "Exchange rate policy in a dollarized economy: a CGE analysis for Bolivia", Kieler Arbeitspapiere, No. 1255.

Shoven, J., y Walley, J. (1984), "Applied general equilibrium models of taxation and international trade: An introduction and survey", Journal of Economic Literature, Vol. 23, No. 3:1007-1051

Shoven, J., y Walley, J. (1992), “Applying general equilibrium”, New York: Cambridge University Press. 
Taylor, L. (1990), "Structuralist CGE Models" en Taylor, L. (ed.), Socially Relevant Policy Analysis: Structuralist Computable General Equilibrium Models for the developing world, Cambridge, MA: MIT Press.

Taylor, L., Sarkar, H., y Rattso, J. (1984), "Macroeconomic adjustment in a general equilibrium model for India", en M. Syrquin, L. Taylor and L. E. Westphal (eds.), Economic Structure and Performance. New York: Academic Press.

Taylor, L., y Black, S. L. (1974), "Practical General Equilibrium Estimation of Resources Pulls under Trade Liberalization", Journal of International Economics, Vol. 4(1): 37-58, April.

Taylor, L., y Lysy, F., (1979), "Vanishing Income Distributions. Keynesian Clues about model surprises in the short run", Journal of Development Economics, Vol. 6: 11-29.

Toporowski, J., y Michell, J. (2011), "The stock-flow consistent approach with active financial markets". http://www.postkeynesian.net/downloads/soas12/JM080612.pdf.

Yeldan, E., Voyvoda, E., y Telli, C. (2008), "Macroeconomics of twin-targetting in Turkey: Analytics of a Financial CGE Model", International Review of Applied Economics, Vol. 22, 2: 227- 242.

Zuleta, H., y Arango, J (1994), "Efectos de una bonanza petrolera a la luz de un modelo de optimización intertemporal", Archivos de Macroeconomía No. 29. Departamento Nacional de Planeación.

\section{Para citaciones:}

Escobar-Espinoza, A. A., Guevara-Castañeda, D. A., \& Uribe-Veloza, M. A. (2017). Modelos Computacionales y Análisis de la Política Económica en Colombia. Panorama Económico, 25, 4, pp. 535-558.

\section{AUTORES}

Andres Escobar-Espinoza

Profesor e Investigador del Programa de Economía de la Facultad de Ciencias Economicas de la Universidad de Cartagena (Colombia). PhD en Economía de la Universita Cattolica del Sacro Cuore (Italia) y Director del Departamento de Investigaciones Económicas y Sociales de la Universidad de Cartagena

Diego Alejandro Guevara-Castañeda

Profesor e Investigador del Programa de Economía de la Universidad Nacional de Colombia (UNAL). PhD y Magister en Ciencias Económicas de la UNAL (Colombia). Cuenta con experiencia en investigación en la University of Limerick (Irlanda) y en el Levy Economics Institute of Bard College. Cuenta con experiencia académica en instituciones como la Universidad de la Sabana, Pontifica Universidad Javeriana, Universidad Católica de Colombia.

\section{Miguel Andrés Uribe Veloza}

Actualmente vinculado a New York University (USA) y cuenta con estudios de posgrado en Análisis y Políticas Económicas en la École des Hautes Études en Sciences Sociales (EHESS), Francia. 